\title{
Asteroseismology of red giants to constrain angular momentum transport
}

\author{
P. Eggenberger \\ Geneva Observatory, University of Geneva, Maillettes 51, 1290, Sauverny, Switzerland \\ email: patrick.eggenberger@unige.ch
}

\begin{abstract}
Asteroseismic data obtained by the Kepler spacecraft have led to the recent detection and characterization of rotational frequency splittings of mixed modes in red-giant stars. This has opened the way to the determination of the core rotation rates for these stars, which is of prime importance to progress in our understanding of internal angular momentum transport. In this contribution, we discuss which constraints can be brought by these asteroseismic measurements on the modelling of angular momentum transport in stellar radiative zones.
\end{abstract}

Keywords. stars: evolution, stars: interiors, stars: oscillations, stars: rotation

\section{Introduction}

The inclusion of rotational effects basically changes all outputs of stellar models (e.g. Maeder 2009). A coherent physical description of rotational effects has to take into account simultaneously the transport of chemical elements and angular momentum. The efficiency of angular momentum transport is then found to have an important impact on the properties of rotating models. To progress in our understanding of internal angular momentum transport, one needs direct observational constraints on the internal rotation of stars, which are now available thanks to seismic data.

Helioseismic measurements indicate a nearly flat rotation profile in the radiative zone of the Sun (Brown et al. 1989; Elsworth et al. 1995; Kosovichev et al. 1997; Couvidat et al. 2003; García et al. 2007), while solar models including only shellular rotation predict a rapidly rotating core (Pinsonneault et al. 1989; Chaboyer et al. 1995; Eggenberger et al. 2005; Turck-Chièze et al. 2010). This is a first indication that an undetermined mechanism for the angular momentum transport is at work in the solar interior.

In the continuity of helioseismic measurements, solar-like oscillations have been observed for a large number of stars. In particular, rotational frequency splittings have been recently detected and characterized for red giants (Beck et al. 2012; Deheuvels et al. 2012; Mosser et al. 2012; Deheuvels et al. 2014). In this contribution, we briefly discuss the implications of these new observational constraints on the modelling of angular momentum transport in stellar radiative zones.

\section{Mixed oscillation modes in the red giant KIC 8366239}

The first detection and characterization of rotational frequency splittings of mixed modes has been obtained for the red giant KIC 8366239 (Beck et al. 2012). These measurements show that oscillation modes at the center of the dipole forests have lower values of rotational splitting than modes in the wings of the dipole forests. This directly indicates that differential rotation is present in the interior of this red giant, with a core rotating more rapidly than the surface (Beck et al. 2012). At first sight, this seems to be in good agreement with stellar models, which predict differential rotation during the 
red-giant phase because of the rapid contraction of the central layers after the main sequence (e.g Palacios et al. 2006; Eggenberger et al. 2010). However, by computing models including rotation for this target and comparing the theoretical values for the rotational splittings with the observed ones, we conclude that rotating models predict too steep internal rotation profiles compared to asteroseismic measurements (Eggenberger et al. 2012; Marques et al. 2013). This clearly indicates that an additional mechanism for the internal angular momentum transport is at work during the post-main sequence phase.

In order to study how this unknown transport mechanism can be constrained by asteroseismic data, Eggenberger et al. (2012) introduced an additional viscosity $\nu_{\text {add }}$ corresponding to this process in the equation describing the internal transport of angular momentum. By computing models of KIC 8366239 for different values of $\nu_{\text {add }}$ and of the initial velocity on the zero-age main sequence (ZAMS), one finds that the mean value for the efficiency of this unknown transport mechanism is strongly constrained by asteroseismic measurements to the value of $\nu_{\text {add }}=3 \cdot 10^{4} \mathrm{~cm}^{2} \mathrm{~s}^{-1}$ for this $1.5 M_{\odot}$ red giant (Eggenberger et al. 2012).

\section{KIC 7341231: mixed modes in a low-mass red giant}

In the preceding section, we have seen that an additional mechanism for the internal transport of angular momentum is needed during the post-main sequence evolution of a $1.5 M_{\odot}$. This star exhibits a convective core during the main sequence and one can then wonder whether such a mechanism is also needed for a low-mass star with a radiative core during the main sequence. Rotational splittings have been obtained for KIC 7341231, a red giant with a mass of about $0.84 M_{\odot}$ (Deheuvels et al. 2012). For this star with a radiative core during the main sequence, one also finds that an efficient additional mechanism for the internal transport of angular momentum is needed in order to correctly reproduce the asteroseismic data (Ceillier et al. 2012, 2013).

As for the more massive red giant KIC 8366239, the efficiency of this unknown transport process can be determined for KIC 7341231 by computing models including an additional viscosity $\nu_{\text {add }}$. In this case, we find a lower value of about $3 \cdot 10^{3} \mathrm{~cm}^{2} \mathrm{~s}^{-1}$ for the additional viscosity. The model of KIC 7341231 has a convective enveloppe during the main sequence and the initial velocity on the ZAMS is thus found to depend on the adopted efficiency for the associated surface magnetic braking during the main sequence. Interestingly, the value of the additional viscosity $\nu_{\text {add }}$ can be precisely determined independently from the uncertainties on the adopted magnetic braking during the main sequence. The differences in $\nu_{\text {add }}$ between KIC 8366239 and KIC 7341231 suggests that the efficiency of the undetermined additional process for the transport of angular momentum decreases when the mass of the star decreases.

\section{Rotational splittings for a sample of six subgiants and young red giants}

Asteroseismic measurements have been recently obtained for a sample of six Kepler young red giants (Deheuvels et al. 2014). Estimates of the core and surface rotation rates have then been deduced for these stars. These results suggest that the degree of differential rotation increases when evolution proceeds, with an increase of the core rotation rate and a decrease of the surface rotation rate when gravity decreases (Deheuvels et al. 2014). 
Interestingly, rotational splittings suggest that for two of these targets a discontinuous internal rotation profile is favored compared to a smooth rotation profile. The determination of the location of such a discontinuity suggests that it corresponds to the location of the hydrogen-burning shell. These results seem to indicate that the efficiency of the additional mechanism of internal angular momentum transport should strongly decrease in a region of large chemical gradients. Such a sharp discontinuity in the rotation profile seems to be difficult to reproduce with fossil magnetic fields and could favor diffusive processes with a reduced efficiency related to the presence of steep chemical gradients.

By comparing the deduced core and surface rotation rates with rotating models including an additional viscosity associated to the unkown transport process, one can precisely determined the efficiency of this process for the six targets. This also suggests an increase of $\nu_{\text {add }}$ with the mass in good agreement with the preceding result obtained by comparing KIC 8366239 and KIC 7341231. Interestingly, the simultaneous determination of the core and surface rotation rates for these stars can also constrain the efficiency of the magnetic braking associated to the presence of a convective envelope during the main-sequence phase. One also finds that the change of the efficiency of the transport of angular momentum in the radiative zone has a negligible impact on the surface rotation rates during the red-giant phase (see dashed lines in Fig. 1). This is obvious in view of the difference in the moment of inertia between the core and the enveloppe of a red giant. It is however interesting to recall this point in the context of red giants exhibiting a rapid surface rotation velocity, which origin is more related to binary interaction or planet engulfment than to an efficient transport of angular momentum in the radiative interior.

\section{Core rotation rates for a large sample of red giants}

Mosser et al. (2012) have determined mean core rotation rates from rotational splittings for a large sample of red giants observed with the Kepler spacecraft. Interestingly, this study suggests that core rotation rates slightly decrease during the evolution on the red-giant branch.

As shown in Fig. 1, a model including only shellular rotation (dotted line) predicts a very rapidly rotating core during the red giant phase. Moreover, it predicts an increase of the core rotation rate when the star evolves on the red-giant branch, which is also in contradiction with asteroseismic data. When an additional constant viscosity of $3 \cdot 10^{4} \mathrm{~cm}^{2} \mathrm{~s}^{-1}$ is included (continuous line in Fig. 1), the model correctly reproduces the asteroseismic data available for the red giant KIC 8366239 and also predicts a slight increase in the core rotation rates of red giants with a radius lower than about $5 R_{\odot}$. This is in good agreement with core rotation rates deduced from asteroseismic data. However, for higher values of the radius, this model predicts an increase of the core rotation rates when evolution proceeds. This discrepancy with the trend deduced from asteroseismic measurements indicates that the efficiency of the unknown transport mechanism of angular momentum must increase when the star evolves along the red-giant branch.

\section{Comparison with mechanisms already included in stellar evolution codes}

In the preceding sections, we have seen that according to asteroseismic data an unknown mechanism is needed in addition to meridional circulation and the shear instability for the transport of angular momentum in the radiative zone of red giants. Asteroseismic data also bring valuable constraints on the efficiency of such a process for red giants of 


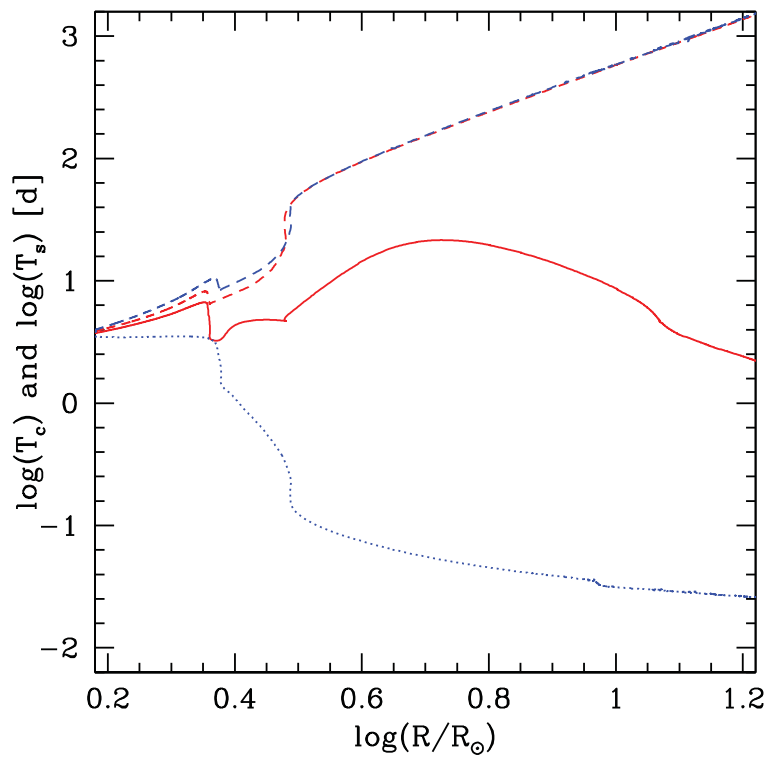

Figure 1. Core (continuous and dotted lines) and surface (dashed lines) rotation periods for two rotating models of $1.5 \mathrm{M}_{\odot}$ with an initial velocity of $20 \mathrm{~km} \mathrm{~s}^{-1}$ on the ZAMS. The dotted line (blue) indicates a model computed with shellular rotation only. The continuous line (red) corresponds to the same model except for the inclusion of an additional viscosity of $3 \times 10^{4} \mathrm{~cm}^{2} \mathrm{~s}^{-1}$.

different masses and at different evolutionary stages. One can then wonder whether these seismic constraints can be correctly reproduced by mechanisms already included in some evolution codes such as magnetic fields and internal gravity waves.

Internal gravity waves are found to be able to efficiently transport angular momentum in the radiative zone of solar-type stars (see e.g. Zahn et al. 1997; Talon \& Charbonnel 2003; Charbonnel \& Talon 2005; Mathis et al. 2013). It would then be interesting to see whether rotating models of red giants including the transport of angular momentum by internal gravity waves are able to correclty reproduce the asteroseismic constraints now available for the internal rotation of red giants.

Concerning magnetic fields, Cantiello et al. (2014) recently compared rotating models of red giants including the Tayler-Spruit dynamo (Spruit 1999, 2002) to asteroseismic measurements. They find that models including the transport of angular momentum by the Tayler-Spruit dynamo are in better agreement with asteroseismic determinations of core rotation rates of red giants than models including only rotational effects. However, models including the Tayler-Spruit dynamo still predict core rotation rates which are at least ten times higher than the observed values.

\section{Summary}

Asteroseismic constraints on the internal rotation of red giants are now available to help us progress in the modelling of the different transport processes at work in stellar radiative zones. The goal is now to use these new valuable constraints to obtain a coherent global physical description of transport processes in radiatives zones that should be valid for low and massive stars, from the pre-main sequence to advanced stages of evolution.

Preliminary results of comparison of these asteroseismic measurements with stellar models clearly show that an undetermined mechanism for the internal transport of angular momentum is needed during the post-main sequence evolution. This is the case for 
low-mass red giants with a radiative core during the main sequence as well as for more massive stars with a convective core during the main sequence. Thanks to seismic data, the efficiency of this unknown transport process can be precisely determined. Asteroseismic measurements suggest an increase of the efficiency of this process when the mass increases as well as when the star evolves on the red-giant branch.

Concerning the physical nature of this unknown process, it will be interesting to compare the rotation profiles of red-giant models including angular momentum transport by internal gravity waves to the asteroseismic constraints now available. Different clues may suggest that transport of angular momentum by magnetic instabilities could be a promising candidate. While a first study of the impact of the Tayler-Spruit dynamo indicates that the efficiency of this process is not sufficient to account for the low core rotation rates deduced from seismic data (Cantiello et al. 2014), it will be particularly interesting to consider the effects of the azimuthal magnetorotational instability in this context (e.g. Rüdiger et al. 2014, and see the contribution by M. Gellert in this volume).

\section{References}

Beck, P. G., Montalban, J., Kallinger, T., et al. 2012, Nature 481, 55

Brown, T. M., Christensen-Dalsgaard, J., Dziembowski, W. A., et al. 1989, ApJ 343, 526

Cantiello, M., Mankovich, C., Bildsten, L., Christensen-Dalsgaard, J., \& Paxton, B. 2014, ApJ 788, 93

Ceillier, T., Eggenberger, P., García, R. A., \& Mathis, S. 2012, Astronomische Nachrichten 333, 971

Ceillier, T., Eggenberger, P., García, R. A., \& Mathis, S. 2013, A\&A 555, A54

Chaboyer, B., Demarque, P., \& Pinsonneault, M. H. 1995, ApJ 441, 865

Charbonnel, C. \& Talon, S. 2005, Science 309, 2189

Couvidat, S., García, R. A., Turck-Chièze, S., et al. 2003, ApJ (Letters) 597, L77

Deheuvels, S., Doğan, G., Goupil, M. J., et al. 2014, A\&A 564, A27

Deheuvels, S., García, R. A., Chaplin, W. J., et al. 2012, ApJ 756, 19

Eggenberger, P., Maeder, A., \& Meynet, G. 2005, A\&SA 440, L9

Eggenberger, P., Miglio, A., Montalban, J., et al. 2010, A\& A 509, A72

Eggenberger, P., Montalbán, J., \& Miglio, A. 2012, A\& A 544, L4

Elsworth, Y., Howe, R., Isaak, G. R., et al. 1995, Nature 376, 669

García, R. A., Turck-Chièze, S., Jiménez-Reyes, S. J., et al. 2007, Science 316, 1591

Kosovichev, A. G., Schou, J., Scherrer, P. H., et al. 1997, Sol. Phys. 170, 43

Maeder, A. 2009, Physics, Formation and Evolution of Rotating Stars, Springer Berlin Heidelberg

Marques, J. P., Goupil, M. J., Lebreton, Y., et al. 2013, A\&A 549, A74

Mathis, S., Decressin, T., Eggenberger, P., \& Charbonnel, C. 2013, A\&A 558, A11

Mosser, B., Goupil, M. J., Belkacem, K., et al. 2012, A\& A 548, A10

Palacios, A., Charbonnel, C., Talon, S., \& Siess, L. 2006, A\&A 453, 261

Pinsonneault, M. H., Kawaler, S. D., Sofia, S., \& Demarque, P. 1989, ApJ 338, 424

Rüdiger, G., Gellert, M., Schultz, M., Hollerbach, R., \& Stefani, F. 2014, MNRAS 438, 271

Spruit, H. C. 1999, A\&A 349, 189

Spruit, H. C. 2002, A\&A 381, 923

Talon, S. \& Charbonnel, C. 2003, A\& A 405, 1025

Turck-Chièze, S., Palacios, A., Marques, J. P., \& Nghiem, P. A. P. 2010, ApJ 715, 1539

Zahn, J.-P., Talon, S., \& Matias, J. 1997, A $\& A$ 322, 320

\section{Discussion}

MoravveJI: Can we take the lessons learned about viscosity on RGB phase to other stallar evolution phases? 
EGGENBERGER: Yes, the idea is to use all available observational constraints to progress in the modelling of transport processes in stellar radiative zones. In this context, the asteroseismic data obtained for red giants are particularly valuable. A coherent physical description of these processes should then be valid for low-mass stars as well as for massive stars and from the pre-main sequence to advanced stages of evolution.

HiRSCHI: Can you confirm that a process transporting angular momentum all the way to the centre of the star is needed to reproduce observations?

EGGENBERGER: Yes, core rotation rates of red giants deduced from asteroseismic measurements clearly show that an efficient process for the transport of angular momentum is needed in addition to meridional circulation and the shear instability to extract angular momentum from the central layers.

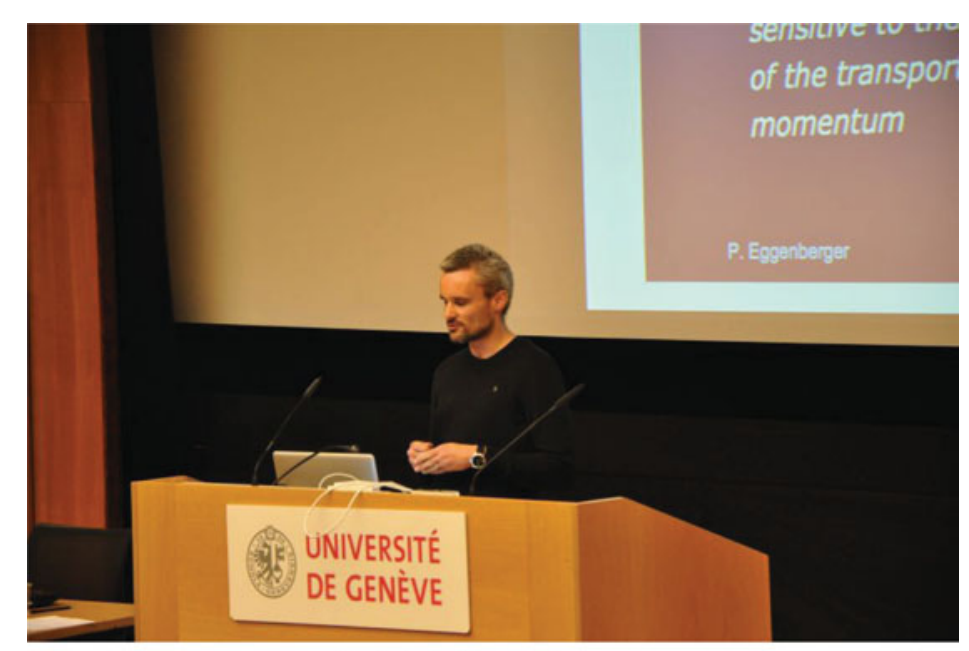

Patrick Eggenberger

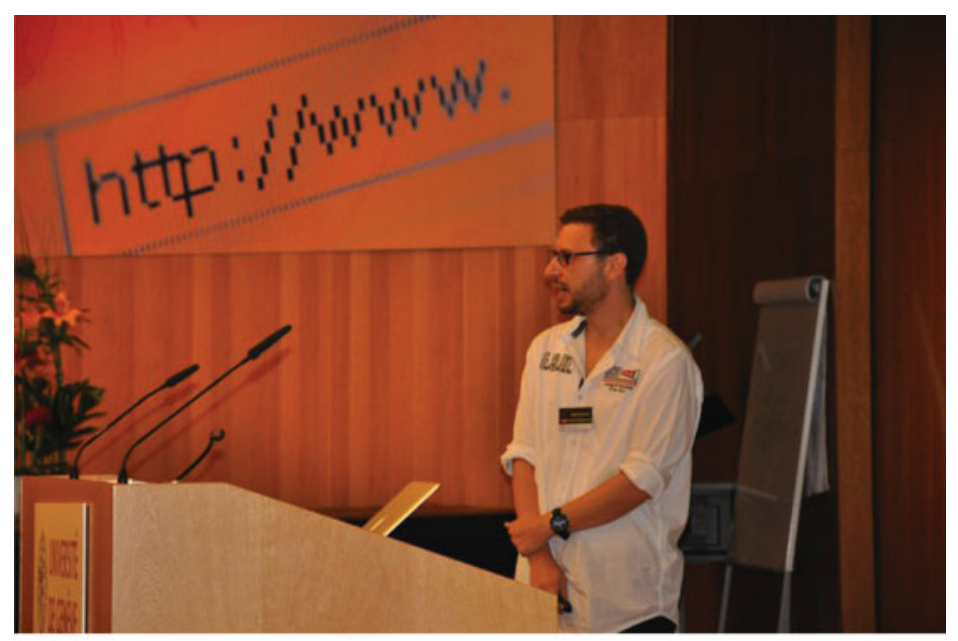

Michalis Kourniotis 